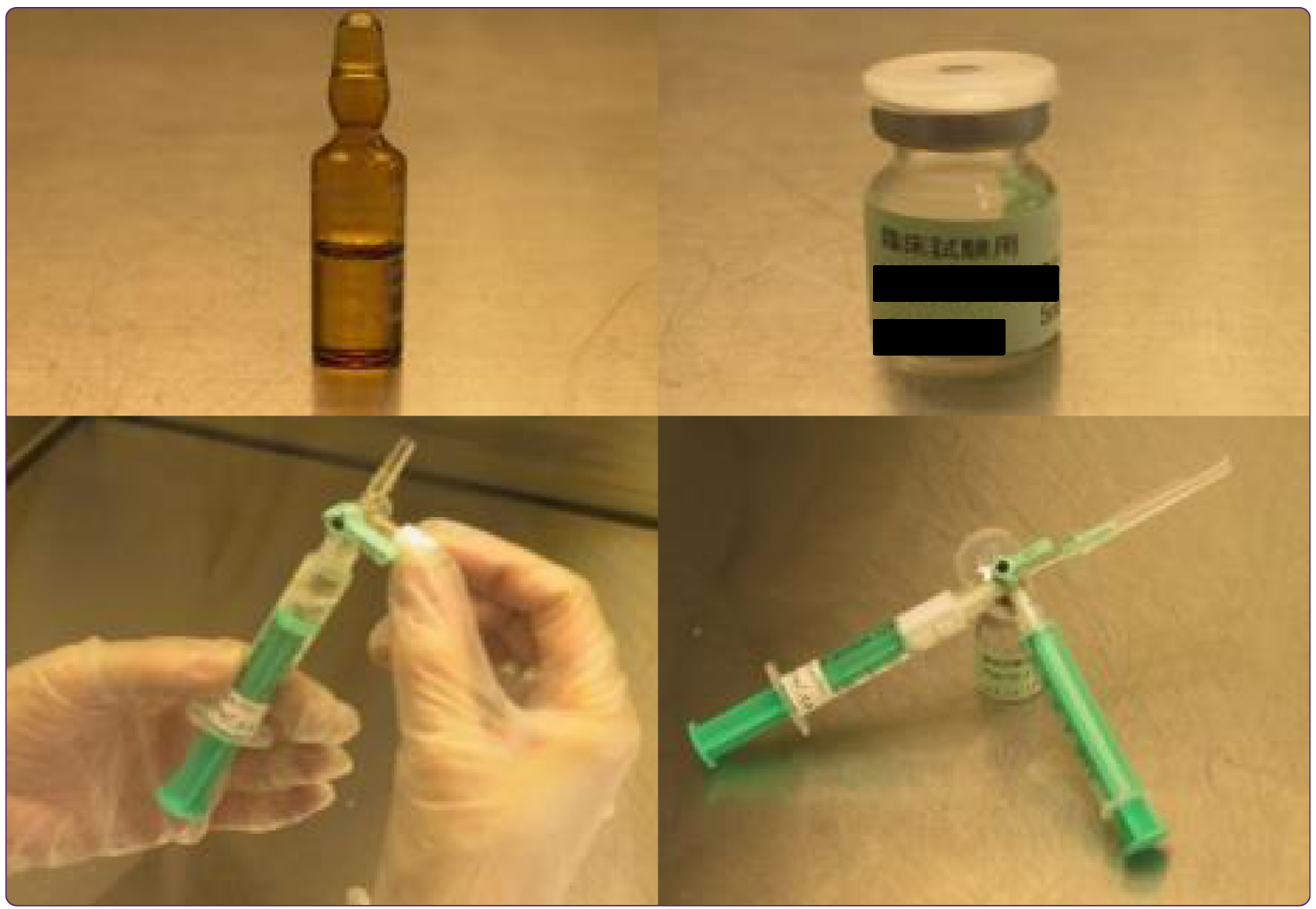

Feasibility study of personalized peptide vaccination for metastatic recurrent triple-negative breast cancer patients

Takahashi et al. 


\title{
Feasibility study of personalized peptide vaccination for metastatic recurrent triple-negative breast cancer patients
}

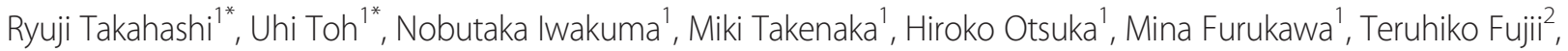
Naoko Seki ${ }^{2}$, Akihiko Kawahara ${ }^{3}$, Masayoshi Kage ${ }^{3}$, Satoko Matsueda ${ }^{4}$, Yoshito Akagi', Akira Yamada², Kyogo Itoh ${ }^{4}$ and Tetsuro Sasada ${ }^{4}$

\begin{abstract}
Introduction: Since treatment modalities for metastatic recurrent triple-negative breast cancer (mrTNBC) are limited, a novel treatment approach including immunotherapy is required. We have developed a novel regimen of personalized peptide vaccination (PPV), in which vaccine antigens are individually selected from a pool of different peptide candidates based on the pre-existing host immunity. Herein we conducted a phase II study of PPV for metastatic recurrent breast cancer patients to investigate the feasibility of PPV for mrTNBC.

Methods: Seventy-nine patients with metastatic recurrent breast cancer who had metastases and had failed standard chemotherapy and/or hormonal therapy were enrolled. They were subgrouped as the mrTNBC group $(n=18)$, the luminal/human epidermal growth factor receptor 2 (HER2)-negative group $(n=41)$ and the HER2-positive group $(n=18)$, while the remaining two patients had not been investigated. A maximum of four human leukocyte antigen (HLA)-matched peptides showing higher peptide-specific immunoglobulin $\mathrm{G}(\mathrm{IgG})$ responses in pre-vaccination plasma were selected from 31 pooled peptide candidates applicable for the four HLA-IA phenotypes (HLA-A2, -A24, or -A26 types, or HLA-A3 supertypes), and were subcutaneously administered weekly for 6 weeks and bi-weekly thereafter. Measurement of peptide-specific cytotoxic T lymphocyte (CTL) and IgG responses along with other laboratory analyses were conducted before and after vaccination.

Results: No severe adverse events associated with PPV were observed in any of the enrolled patients. Boosting of CTL and/or lgG responses was observed in most of the patients after vaccination, irrespective of the breast cancer subtypes. There were three complete response cases (1 mrTNBC and 2 luminal/HER2-negative types) and six partial response cases (1 mrTNBC and 5 luminal/HER2-negative types). The median progression-free survival time and median overall survival time of mrTNBC patients were 7.5 and 11.1 months, while those of luminal/HER2-negative patients were 12.2 and 26.5 months, and those of HER2-positive patients were 4.5 and 14.9 months, respectively.
\end{abstract}

Conclusions: PPV could be feasible for mrTNBC patients because of the safety, immune responses, and possible clinical benefits.

Clinical Trial Registration Number: UMIN000001844 (Registration Date: April 5, 2009)

\footnotetext{
* Correspondence: takahashi_ryuuji@med.kurume-u.ac.jp;

utoh@med.kurume-u.ac.jp

'Department of Surgery, Kurume University School of Medicine, 67

Asahi-machi, Kurume 830-0011, Japan

Full list of author information is available at the end of the article
} 


\section{Introduction}

Recent advances in chemotherapies, hormonal therapies and anti-human epidermal growth factor receptor 2 (HER2) therapies have significantly improved the prognosis in metastatic recurrent breast cancer patients. For example, new chemotherapies using agents such as nanoparticle albumin-bound paclitaxel (nab-PTX) $[1,2]$, eribulin mesylate $[3,4]$ and bevacizumab [5-7], new hormonal therapies such as fluvestrant injection $[8,9]$ or new anti-HER2 therapies such as those using pertuzumab $[10,11]$ and trastuzumab emtansine (T-DM1) [12] have shown significant clinical benefits in metastatic recurrent breast cancer patients. Despite these novel therapeutic advances, the treatment modalities for chemotherapyresistant triple-negative breast cancer (TNBC) remain limited, and thus a novel treatment approach including immunotherapy is required. Nevertheless, no randomized controlled trials of cancer vaccine have shown promise of clinical benefit for metastatic recurrent breast cancer patients, particularly in metastatic recurrent TNBC (mrTNBC).

We have developed a novel regimen of personalized peptide vaccination (PPV), in which vaccine antigens are selected from a pool of 31 different peptide candidates based on the pre-existing immunoglobulin G (IgG) responses specific to each peptide before vaccination [13-17]. Most of the peptides employed for PPV, except for those derived from prostate-related antigens, are known to be commonly expressed in various types of advanced cancers. Our previous clinical trials of PPV for patients with advanced cancers demonstrated the safety and feasibility of this new approach [13-17]. Here we conducted a phase II study of PPV for metastatic recurrent breast cancer to investigate the feasibility of PPV for mrTNBC.

\section{Methods}

\section{Patients and methods}

Women with a histological diagnosis of metastatic recurrent breast cancer were eligible for inclusion in the present study. All patients were required to have evaluable recurrent and/or metastatic tumors at the time of entry. Patients were divided into three different intrinsic subtypes as follows: luminal (estrogen-receptor-positive)/ HER2-negative type, HER2-positive type (immunohistochemical score $3+$ or HER2 gene/chromosome 17 ratio $>2.2$ in fluorescence in situ hybridization) and TNBC (hormone-receptor-negative and HER2-negative). Most patients had failed standard chemotherapy, but a few patients who had failed hormonal therapy alone were also eligible for this study. All patients were required to show positive IgG responses to at least 2 of the 31 different vaccine candidate peptides, as reported previously [13-17]. Other inclusion criteria were as follows: age between 20 and 80 years; an Eastern Cooperative Oncology
Group (ECOG) performance status of 0 or 1; positive status for human leukocyte antigen (HLA)-A2, -A24 or -A26 types, or HLA-A3 supertypes (A3, A11, A31 or A33); life expectancy of at least 12 weeks; and adequate hematologic, hepatic and renal function. Exclusion criteria included pulmonary, cardiac or other systemic diseases; an acute infection; a history of severe allergic reactions; pregnancy or nursing; and other inappropriate conditions for enrollment as judged by clinicians. Patients with a lymphocyte count of $<1,000 / \mu \mathrm{L}$ were excluded from the study, since we previously reported that pre-vaccination lymphocytopenia $(<1,000$ cells $/ \mu \mathrm{L})$ is an unfavorable factor for overall survival (OS) in cancer patients receiving PPV [17]. The protocol was approved by the Kurume University Ethical Committee and registered in the UMIN Clinical Trials Registry (Registration, number UMIN000001844; Registration date, 5 April 2009). All patients were given a full explanation of the protocol and provided their informed consent before enrollment.

\section{Clinical protocol}

This was a phase II study to evaluate the safety and immunological responses in metastatic recurrent breast cancer patients under PPV. Thirty-one peptides, whose safety and immunological effects for other types of cancer were confirmed in previously conducted clinical studies [14-17], were employed for vaccination (12 peptides for HLA-A2, 16 peptides for HLA-A24, 9 peptides for HLA-A3 supertypes (-A3, -A11, -A31, and -A33) and 4 peptides for HLA-A26) (Additional file 1: Table S1). These peptides were prepared under the conditions of Good Manufacturing Practice (GMP) by the PolyPeptide Laboratories (San Diego, CA, USA) and American Peptide Company (Vista, CA, USA). Peptides for vaccination of individual patients were selected in consideration of the pre-existing host immunity before vaccination, as assessed by the titers of IgG specific to each of the 31 different vaccine candidates.

A maximum of four peptides ( $3 \mathrm{mg} /$ each peptide), which were selected based on the results of HLA typing and peptide-specific IgG titers, were subcutaneously administered with incomplete Freund's adjuvant (Montanide ISA51; Seppic, Paris, France) once a week for six consecutive weeks. After the first cycle of six vaccinations, up to four antigen peptides, which were re-selected according to the titers of peptide-specific IgG at the sixth vaccination, were administered every two weeks. After the second cycle of six vaccinations, up to four antigen peptides, which were also re-selected, were administered every four to eight weeks according to the immune responses after PPV. These protocols were continued until remarkable disease progression or disease in remission was shown, according to the will of the individual patient. During the PPV, patients were allowed to receive combination therapies 
such as chemotherapy, hormonal therapy, anti-HER2 therapy and radiotherapy. Adverse events were monitored according to the National Cancer Institute Common Terminology Criteria for Adverse Events version 3.0 (NCI-CTC Ver.-3.0). Complete blood counts and serum biochemistry tests were performed after every six vaccinations. The clinical responses were determined by the Response Evaluation Criteria in Solid Tumors (RECIST) in the vaccinated patients. The RECIST-based clinical responses were evaluated after nearly 12 vaccinations by radiological findings of computed tomography $(\mathrm{CT})$ scan and/or magnetic resonance imaging (MRI), and the best overall responses during PPV treatment were shown. For the patients who did not complete the second cycle of 12 vaccinations, the newest radiological findings were evaluated, except in the case of patients who had died before the RECIST-based radiological evaluation.

Measurement of humoral and cellular immune responses Humoral immune responses specific to each of the 31 peptide candidates were determined by peptide-specific IgG levels using the Luminex system (Luminex, Austin, TX, USA), as previously reported [18]. If the titers of peptidespecific IgG to at least one of the vaccinated peptides in the post-vaccination plasma were more than two-fold higher than those in the pre-vaccination plasma, the changes were considered to be significant, as previously reported [14-17]. Cellular immune responses specific to the vaccinated peptides were evaluated by interferon (INF)- $\gamma$ ELISPOT using peripheral blood mononuclear cells (PBMCs) as previously

Table 1 Comparison of patient characteristics for overall and breast cancer subtypes

\begin{tabular}{|c|c|c|c|c|c|}
\hline Character & $\begin{array}{c}\text { Overall } \\
\text { (number }=79 \text { ) }\end{array}$ & $\begin{array}{c}\text { mrTNBC } \\
\text { (number }=18 \text { ) }\end{array}$ & $\begin{array}{l}\text { Luminal/HER2-negative } \\
\text { (number }=41 \text { ) }\end{array}$ & $\begin{array}{l}\text { HER2-positive } \\
\text { (number = 18) }\end{array}$ & $P$-value ${ }^{\mathrm{a}}$ \\
\hline Median age (range) & 57 (30 to 77$)$ & 55 (30 to 65$)$ & 55 (39 to 76$)$ & 62 (51 to 70$)$ & 0.019 \\
\hline Performance status & & & & & 0.046 \\
\hline $0 / 1$ & $58 / 21$ & $14 / 4$ & $32 / 9$ & $11 / 7$ & \\
\hline $\begin{array}{l}\text { Median time to the first PPV from recurrence, } \\
\text { months (range) }\end{array}$ & $35(1.2$ to 165$)$ & 8 (1.2 to 99$)$ & 40 (2 to 123$)$ & 55 (19 to 165$)$ & 0.101 \\
\hline Histopathology & & & & & 0.079 \\
\hline Ductal carcinoma & 71 & 16 & 37 & 17 & \\
\hline Lobular carcinoma & 4 & 1 & 2 & 1 & \\
\hline Others & 4 & 1 & 2 & & \\
\hline Positive status of HLA-A24 & 55 & 10 & 29 & 14 & 0.039 \\
\hline Positive status of HLA-A2 & 22 & 6 & 8 & 8 & 0.015 \\
\hline Visceral metastasis & & & & & 0.009 \\
\hline Yes/No & $60 / 19$ & $8 / 6$ & $29 / 11$ & $16 / 2$ & \\
\hline Brain metastasis & & & & & 0.002 \\
\hline Yes/No & $11 / 68$ & $3 / 11$ & $2 / 38$ & $6 / 12$ & \\
\hline $\begin{array}{l}\text { Median duration of previous chemotherapies, } \\
\text { months (range) }\end{array}$ & 12 (2 to 148$)$ & 9 (4 to 43$)$ & 12 (2 to 9$)$ & 37 (10 to 148$)$ & $<0.0001$ \\
\hline \multicolumn{6}{|l|}{ Usage of previous standard chemotherapy } \\
\hline Anthracycline & 50 & 16 & 22 & 12 & 0.003 \\
\hline Taxane & 58 & 16 & 24 & 17 & 0.0006 \\
\hline Trastuzumab & 18 & & 3 & 15 & $<0.0001$ \\
\hline Regimen number of previous chemotherapies & & & & & 0.008 \\
\hline$<4 / \geq 4$ & $36 / 36$ & $8 / 9$ & $22 / 14$ & $6 / 12$ & \\
\hline \multicolumn{6}{|l|}{ Combined therapies } \\
\hline Oral chemotherapy & 19 & 5 & 8 & 6 & 0.060 \\
\hline Infusion chemotherapy & 32 & 10 & 15 & 7 & 0.038 \\
\hline Anti-HER2 therapy & 11 & & & 11 & $<0.0001$ \\
\hline Hormonal therapy & 23 & 2 & 17 & 4 & 0.006 \\
\hline Median times of peptide vaccination & 14 (2 to 39$)$ & 12 (2 to 30$)$ & 15 (4 to 39$)$ & 12 (6 to 22$)$ & 0.021 \\
\hline
\end{tabular}

${ }^{a}$ The Mann-Whitney $U$ test and Fisher-Freeman-Halton exact test were performed to examine $P$-values for continuous values and categorical values. HER2, human epidermal growth factor 2; HLA, human leukocyte antigens; mrTNBC, metastatic recurrent triple-negative breast cancer; PPV, personalized peptide vaccination. 
Table 2 Adverse events during the PPV

\begin{tabular}{|c|c|c|c|c|}
\hline Adverse event & Grade 1 & Grade 2 & Grade 3 & Grade 4 \\
\hline Injection site reaction & 42 & 37 & & \\
\hline \multicolumn{5}{|l|}{ Constitutional symptom } \\
\hline Fever & 8 & 1 & & \\
\hline Malaise & 7 & 3 & & \\
\hline Edema limbs & 2 & 3 & & \\
\hline Pain & 5 & & & \\
\hline Tumor pain & 4 & 9 & & \\
\hline \multicolumn{5}{|l|}{ Gastrointestinal } \\
\hline Nausea & 4 & & & \\
\hline Mucositis oral & & 1 & & \\
\hline Abdominal pain & & & 1 & \\
\hline Constipation & 1 & 1 & & \\
\hline Diarrhea & 2 & & & \\
\hline \multicolumn{5}{|l|}{ Respiratory } \\
\hline Dyspnea & 5 & 1 & 1 & \\
\hline Cough & 5 & & & \\
\hline Hoarseness & 2 & & & \\
\hline Pneumonitis & & 1 & & \\
\hline Pleural effusion & 1 & & & \\
\hline Hypoxia & & 1 & 2 & \\
\hline \multicolumn{5}{|l|}{ Neurological } \\
\hline Headache & 1 & 1 & & \\
\hline Dysgeusia & 1 & & & \\
\hline Dizziness & 2 & 1 & & \\
\hline $\begin{array}{l}\text { Peripheral sensory } \\
\text { neuropathy }\end{array}$ & 8 & 2 & & \\
\hline $\begin{array}{l}\text { Peripheral motor } \\
\text { neuropathy }\end{array}$ & 1 & & & \\
\hline \multicolumn{5}{|l|}{ Endocrine disorder } \\
\hline Hypothyroidism & & 1 & & \\
\hline \multicolumn{5}{|l|}{ Skin and subcutaneous } \\
\hline Pruritis & 33 & 3 & & \\
\hline Urticaria & 4 & 1 & & \\
\hline \multicolumn{5}{|l|}{ Reproductive system } \\
\hline Vaginal hemorrhage & 2 & & & \\
\hline \multicolumn{5}{|l|}{ Vascular disorders } \\
\hline Hot flashes & 1 & & & \\
\hline Lymphedema & & 1 & & \\
\hline Hypertension & & 1 & & \\
\hline \multicolumn{5}{|l|}{ Blood/Bone marrow } \\
\hline Anemia & 18 & 9 & 2 & 2 \\
\hline Hemoglobin increased & 1 & & & \\
\hline Leukocytopenia & 22 & 11 & 4 & \\
\hline Neutropenia & 2 & 6 & 5 & 1 \\
\hline
\end{tabular}

Table 2 Adverse events during the PPV (Continued)

\begin{tabular}{lccc}
\hline Lymphocytopenia & 29 & 7 & 12 \\
Thrombocytopenia & 6 & 1 \\
Metabolism and nutrition & & & \\
Anorexia & 2 & & \\
Hyponatremia & 1 & & \\
Hyperkalemia & 3 & & \\
Hypocalcemia & 2 & & \\
Hyperglycemia & 2 & & 2 \\
Laboratory & & & 2 \\
AST increased & 14 & 9 & 2 \\
ALT increased & 19 & 4 & 1 \\
Y-GTP increased & 7 & 3 & 1 \\
ALP increased & 6 & 1 & 1 \\
Hyperbilirubinemia & 3 & 1 & 1 \\
Creatinine increased & 10 & 3 & \\
Cholesterol high & 4 & & \\
Hypoalbuminemia & 46 & 7 & 1 \\
INR increased & 1 & 1 & \\
APTT increased & 1 &
\end{tabular}

ALP, alkaline phosphatase; ALT, alanine aminotransferase; AST, aspartate aminotransferase; APTT, activated partial thromboplastin time; $\gamma$-GTP, gamma-glutamyl transpeptidase; INR, international normalized ratio; PPV, personalized peptide vaccination.

reported [14-17]. As a control, cellular immune responses specific to CEF peptides (MABTECH, Cincinnati, OH, USA), a mixture of virus-derived cytotoxic $\mathrm{T}$ lymphocyte (CTL) epitopes, were also examined.

\section{Statistical analyses}

The Mann-Whitney U test and Fisher-Freeman-Halton exact test were used to examine statistical differences for continuous values and categorical values, respectively. $P$-values less than 0.05 were considered to be statistically significant. Progression-free survival (PFS) or OS was calculated from the date of the first vaccination until the date of disease progression or death, respectively, or the last date when the patient was known to be alive. The survival analysis was performed using the Kaplan-Meier method, and a comparison of the survival curves was performed with the log-rank test. Statistical tests were performed using JMP version 10 (SAS Institute Inc., Cary, NC, USA) and StatXact version 8 (Cytel Inc., Cambridge, MA, USA).

\section{Results}

\section{Patient characteristics}

Between January 2009 and April 2013, 79 metastatic recurrent breast cancer patients were enrolled in this study. The patient characteristics are shown in Table 1 
for the overall patient group and each of the three subtypes. Among the 79 patients, 77 patients had been investigated to determine their intrinsic subtype before vaccination, while the remaining 2 patients had not. The HER2-positive group was associated with older median age $(P=0.019)$, restricted performance status $(P=0.046)$, higher positivity of HLA-A24 or -A2 $(P=0.039$ or $P=0.015)$, higher frequency of visceral or brain metastasis $(P=0.009$ or $P=0.002)$ and longer duration of previous chemotherapies $(P<0.0001)$. Although the mrTNBC group had a shorter duration of previous chemotherapies $(P<0.0001)$, most of the mrTNBC patients had received previous standard chemotherapy (anthracycline, $P=0.003$; taxane, $P=0.0006$ ).

\section{Combined therapies and adverse events}

The median number of peptide vaccinations was 14 , with a range from 2 to 39 vaccinations (Table 1 ). Table 2 shows all adverse events during the PPV. As the vaccination-related adverse events, all patients showed grade 1 or 2 dermatological reactions to PPV at the injection sites, but no patients showed severe adverse events (grade 3 or more). Forty-one patients (52.0\%) showed grade 3 or 4 adverse events strongly associated with combined chemotherapies and disease progression (Table 2).

During the PPV, 51 patients (64.6\%; 32 cases with infusion chemotherapy and 19 cases with oral chemotherapy) received combined chemotherapies, while 23 patients (29.1\%) and 11 patients (13.9\%) received hormonal therapies and anti-HER2 therapies, respectively (Table 1). The mrTNBC patients received combined infusion chemotherapy more frequently than other breast cancer subtypes $(P=0.038)$. The most commonly used chemotherapy drug was capecitabine (fifteen cases; $19.0 \%$ ), followed by gemcitabine (eight cases; $10.1 \%$ ), eribulin mesylate (six cases; 7.6\%), FEC (5-fluorouracil, epirubicin and cyclophosphamide), nab-PTX or vinorelbine (four cases each; 5.1\%), paclitaxel (three cases; $3.8 \%$ ), bevacizumab, irinotecan or S-1 (two cases each; $2.5 \%$ ), and docetaxel, oral cyclophosphamide or tegafur (one case each; 1.3\%). Eleven patients (13.9\%) received combined anti-HER2 therapies including trastuzumab (six cases; 7.6\%) and lapatinib (five cases; 6.3\%); combined anti-HER2 therapy was the most used treatment for the HER2-positive group $(P<0.0001)$. In addition, 23 patients $(29.1 \%)$ received combined hormonal therapies using agents, such as aromatase inhibitor (16 cases; 20.3\%), high-dose toremifene (5 cases; 6.3\%) and (a)

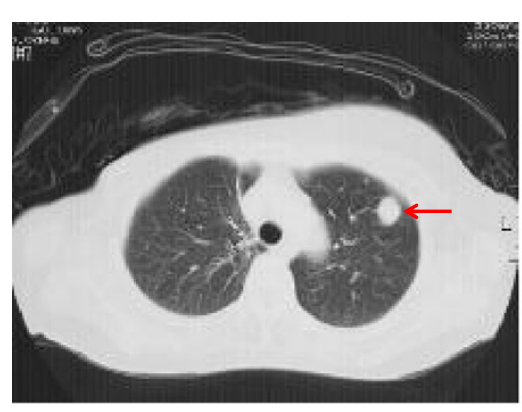

(c)

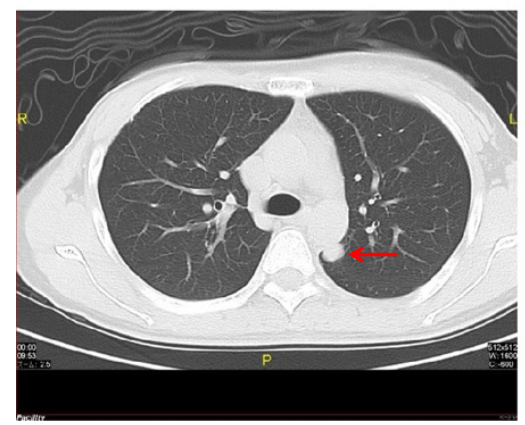

(b)

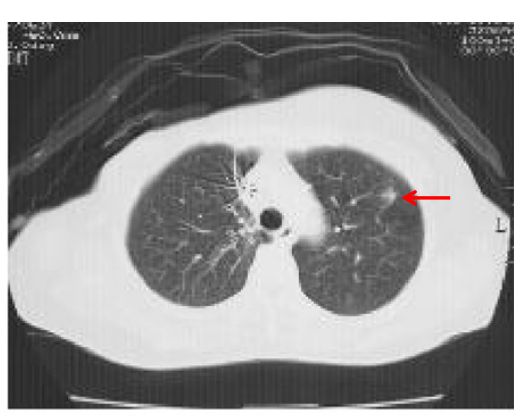

(d)

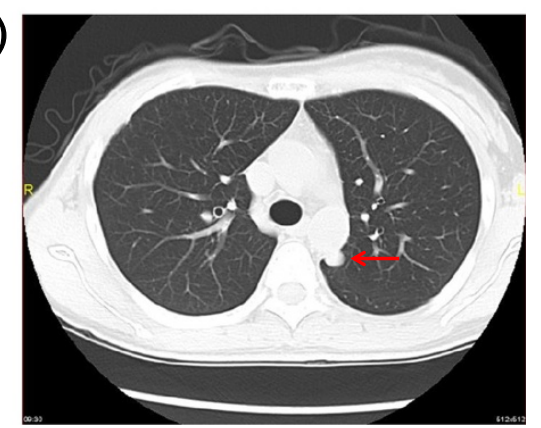

Figure 1 Clinical responses to PPV. $\mathbf{a}, \mathbf{b}$ ) Computed tomography findings of a PR case with mrTNBC (case 2 in Table S2) before and after the 12th vaccination. A 63-year-old woman with a recurrent lung mass underwent 12 vaccinations in combination with gemcitabine $\left(1,000 \mathrm{mg} / \mathrm{m}^{2} /\right.$ week for three weeks followed by one week intermission). At four months after the first vaccination, the lung mass was remarkably reduced in size (arrow). c, d) Computed tomography findings of a SD case with mrTNBC (case 18 in Table S2) before and after the eighth vaccination. A 34-year-old woman with a recurrent lung mass underwent eight vaccinations in combination with two cycles of eribulin mesylate $\left(1.4 \mathrm{mg} / \mathrm{m}^{2} /\right.$ week for two weeks followed by one week intermission). At three months after the first vaccination, the lung mass was slightly decreased in size (arrow). mrTNBC, metastatic recurrent triple negative breast cancer; PPV, personalized peptide vaccination; PR, partial response; SD, stable disease. 
fluvestrant (2 cases; $2.5 \%$ ); combined hormonal therapy was the most used treatment for the luminal/HER2negative group $(P=0.046)$.

\section{Immune responses to the vaccinated peptides}

Both humoral and cellular immune responses specific to the vaccinated peptides were analyzed in blood samples before and after vaccination. Plasma samples were collected from 79,75 , or 53 patients before vaccination, at the 6 th vaccination, or at the 12 th vaccination, respectively. For the monitoring of humoral immune responses, peptide-specific IgGs reactive to each of the 31 different peptides, including both vaccinated and non-vaccinated peptides, were measured by bead-based multiplex assay. The numbers of peptides employed for the first cycle of vaccinations were 2,3 , or 4 in 8,6 , or 63 patients, respectively (Additional file 2: Table S2, Additional file 3: Table S3, and Additional file 4: Table S4). Augmentation of IgG responses specific to at least one of the vaccinated peptides after 6 or 12 vaccinations was observed in 53/75 (70.7\%) patients or 50/53 (94.3\%) patients, respectively. Peptidespecific IgG responses after 6 or 12 vaccinations were augmented in $7 / 15(46.7 \%)$ patients or $9 / 10(90 \%)$ patients with mrTNBC (Additional file 2: Table S2). Such augmentation was seen in $28 / 40(70.0 \%)$ patients or $29 / 31$ (93.5\%) patients in the luminal/HER2-negative group (Additional file 3: Table S3) and in 16/18 (88.9\%) patients or 11/11 $(100 \%)$ patients of the HER2-positive group (Additional file 4: Table S4), respectively.

Cellular immune responses to vaccinated peptides were assessed by IFN- $\gamma$ ELISPOT assay. Antigen-specific CTL responses were detectable in 17/66 (25.8\%) patients before vaccination (Additional file 2: Table S2, Additional file 3: Table S3 and Additional file 4: Table S4). In contrast, augmentation of the CTL responses specific to at least one of the vaccinated peptides after six vaccinations was observed in 34/63 patients (54.0\%). Peptide-specific CTL responses after six vaccinations were augmented in 7/14 (50.0\%) patients with mrTNBC (Additional file 2: Table S2), while such augmentation was seen in 18/31 (58.1\%) patients and 7/16 (43.8\%) patients in the luminal/HER2negative group (Additional file 3: Table S3) and HER2positive group (Additional file 4: Table S4), respectively. We also tested CTL responses to CEF peptides, a mixture of virus-derived CTL epitopes, as a control. CTL responses to CEF peptides were observed in 27/62 (43.5\%) patients before vaccination and 15/58 (25.9\%) patients after six vaccinations (Additional file 2: Table S2, Additional file 3: Table S3 and Additional file 4: Table S4). (a)

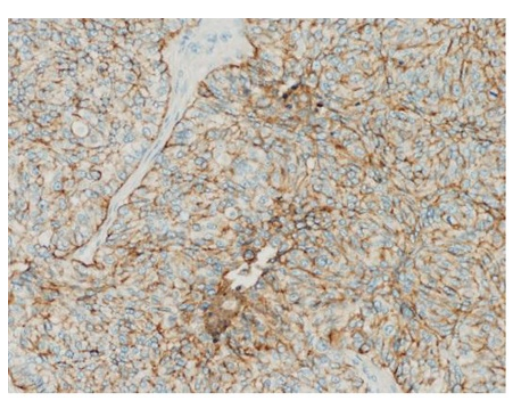

(c)

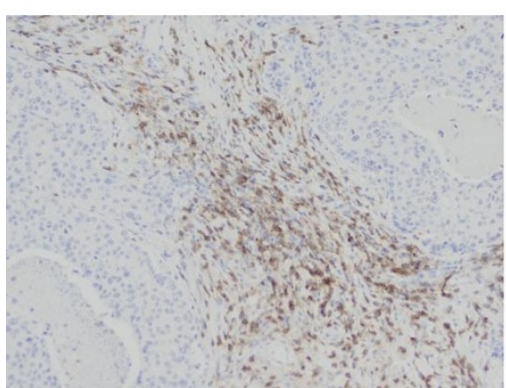

(b)

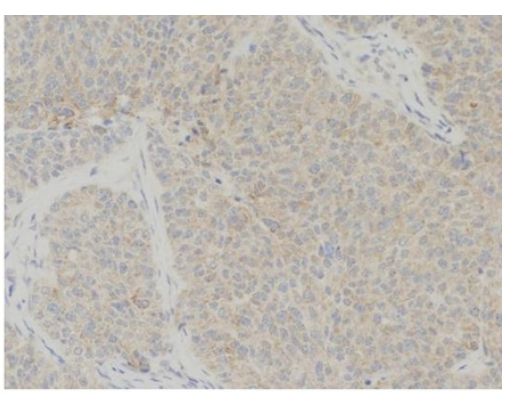

(d)

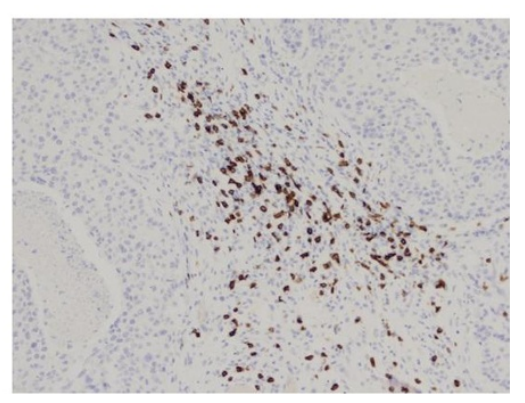

Figure 2 Expressions of TAAs and pathological responses to PPV. After completion of eight vaccinations in combination with two cycles of eribulin mesylate $\left(1.4 \mathrm{mg} / \mathrm{m}^{2} /\right.$ week for two weeks followed by one week intermission), the lung metastasis of a SD case with mrTNBC (case 18 in Table S2) was resected at three months after the first vaccination. The TAA expression and T cell infiltration in the resected lung tissue were examined by immunohistochemistry. a, b) Among the four TAAs, that is, SART2, PSA, EGF-R and LCK, two TAAs were expressed in the lung tumor. a) EGF-R (X200); b) SART2 (X200). c, d) Peritumoral infiltration of T lymphocytes was confirmed in the lung tumor. c) CD4 ${ }^{+}$T lymphocytes (X200); d) CD8+ T lymphocytes (X200). EGF-R, epidermal growth factor receptor; LCK, lymphocyte specific protein tyrosine kinase; mrTNBC, metastatic recurrent triple negative breast cancer; PPV, personalized peptide vaccination; PSA, prostate specific antigen; SART2, squamous cell carcinoma antigen recognized by T-cells 2; SD, stable disease; TAA, tumor associated antigens. 
Collectively, 30/63 (47.6\%) patients showed both increased CTL and IgG responses to the vaccinated peptides, 23/63 (36.5\%) patients showed either increased CTL or IgG responses, and the remaining $10(15.9 \%)$ patients showed neither CTL nor IgG boosting. In patients treated with PPV alone $(n=27)$, IgG responses were more frequently increased than those in patients treated with combined chemotherapies $(\mathrm{n}=47)(P=0.002)$, although there was no significant difference in the increase in CTL responses $(P=1.000)$.

\section{Clinical responses to PPV}

The RECIST-based clinical responses were evaluated in 64 patients by radiological findings. There were 3 complete response (CR), 6 partial response (PR), 27 stable disease (SD) and 28 progressive disease (PD). The overall response rate of PPV was $14 \%$, including three CR and six PR cases. Among the responsive patients, combined chemotherapy was used in eight cases and hormonal therapy in one case. The intrinsic subtypes showed one mrTNBC and two luminal/HER2-negative types in the $\mathrm{CR}$ cases and one mrTNBC and five luminal/HER2-negative types in the PR cases. Computed tomography findings of each of the mrTNBC cases showing PR or SD are shown in Figure 1. The PR case (case 2 in Additional file 2: Table S2) was a 63-year-old woman with a recurrent lung mass treated with a combination of gemcitabine and PPV. At four months after the first vaccination, the lung mass was remarkably reduced in size (Figure 1a and Figure 1b). She survived 32 months after the first vaccination and died due to disease progression. The SD case (case 18 in Additional file 2: Table S2) was a 34-year-old woman with a recurrent lung mass treated with a combination of eribulin mesylate and PPV. At three months after the first vaccination, the lung mass was slightly decreased in size (Figure 1c and Figure 1d). She was subsequently treated by radical resection of the lung mass and pathological evaluation. The lung mass was metastatic TNBC with a high Ki-67 labeling index (42.0\%). It expressed epidermal growth factor receptor (EGF-R) and squamous cell carcinoma antigen recognized by T-cells 2 (SART2) antigens which were vaccinated antigens (Figure 2a and Figure 2b), and peritumoral infiltration of $\mathrm{T}$ lymphocytes was confirmed (Figure 2c and Figure 2d). She is still alive at 13 months following the first vaccination.

\section{Survival analyses by intrinsic subtypes}

Figure 3 shows survival curves for the three intrinsic subtypes. The median progression-free survival time (MPFST) and median overall survival time (MST) of mrTNBC patients were 7.5 and 11.1 months, while those of luminal/ HER2-negative patients were 12.2 and 26.5 months, and those of HER2-positive patients were 4.5 and 14.9 months, respectively. For each intrinsic subtype, the survival curves

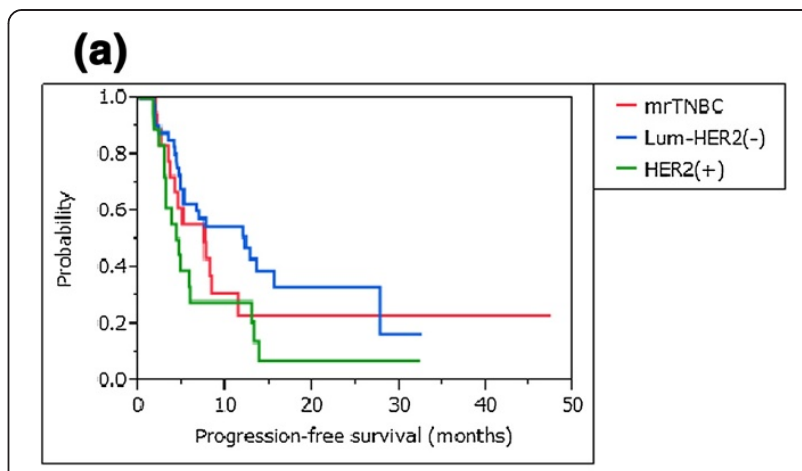

\section{(b)}

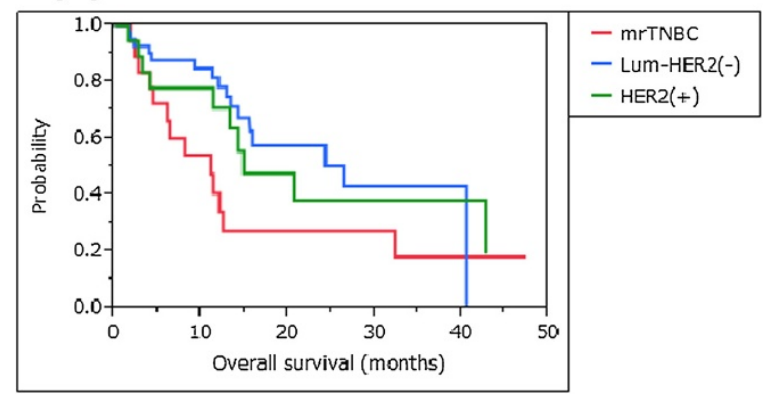

Figure 3 Survival curves among the three intrinsic subtypes. a) The median progression-free survival time of mrTNBC patients was 7.5 months, while that of luminal/HER2-negative patients was 12.2 months, and that of HER2-positive patients was 4.5 months. b) The median overall survival time of mrTNBC patients was 11.1 months, while that of luminal/HER2-negative patients was 26.5 months, and that of HER2-positive patients was 14.9 months. HER2, human epidermal growth factor receptor 2; mrTNBC, metastatic recurrent triple negative breast cancer.

were compared for patients treated with PPV plus concurrent chemotherapies and those treated with PPV alone (Figure 4). There was no significant survival advantage of concurrent chemotherapies in each intrinsic subtype, compared with treatment with PPV alone (mrTNBC: PFS and OS, $P=0.467$ and $P=0.347$, respectively; luminal/HER2negative type: PFS and OS, $P=0.220$ and $P=0.850$, respectively; and HER2-positive type: PFS and OS, $P=0.296$ and $P=0.957$, respectively).

Survival analyses along with analyses of the immune responses to PPV were also conducted in the three subtypes. Figure 5 shows the survival curves for patients with or without increased IgG responses after PPV in each intrinsic subtype. IgG boosting was a significant prognostic factor for OS and PFS in HER2-positive patients, whereas there was no significant difference between increased IgG responses and these prognoses in mrTNBC and luminal/ HER2-negative patients (mrTNBC: PFS and OS, $P=0.274$ and $P=0.152$, respectively; luminal/HER2-negative type: PFS and OS, $P=0.732$ and $P=0.571$, respectively; HER2positive type: PFS and OS, $P=0.0001$ and $P=0.001$, respectively). Figure 6 shows the survival curves for patients 


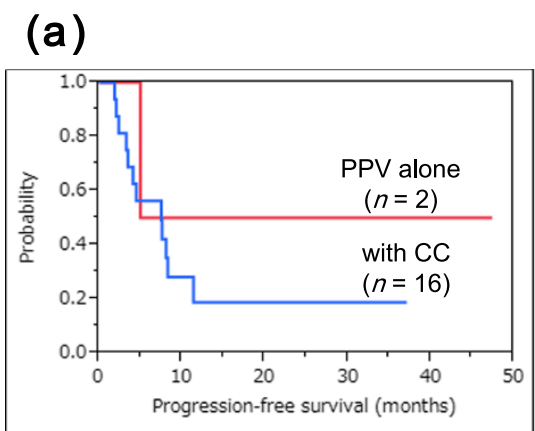

mrTNBC

(b)

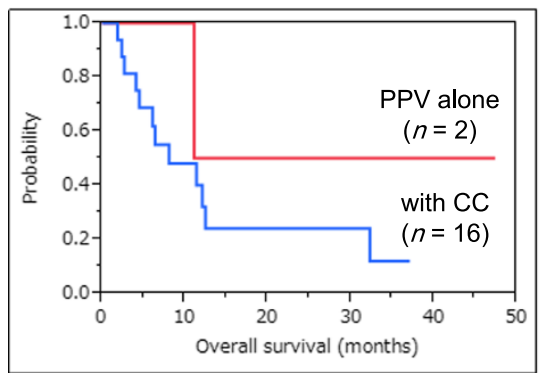

(c)

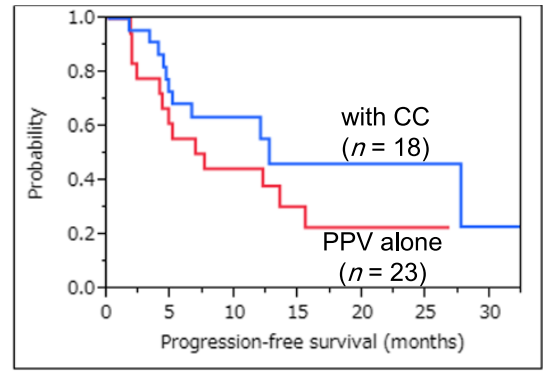

Lum-HER2(-)

(d)

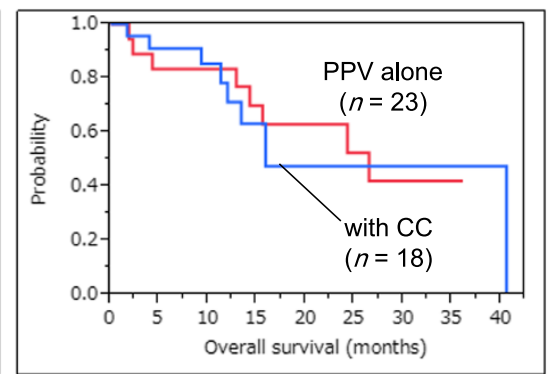

(e)

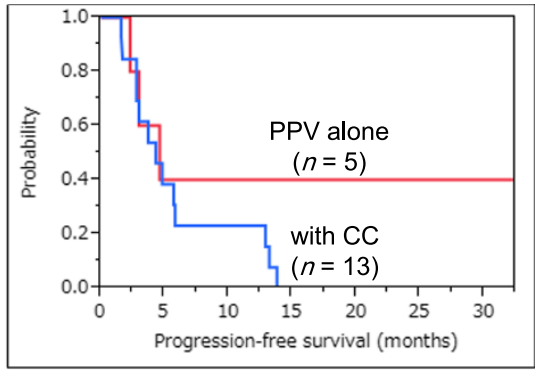

HER2(+)

Figure 4 Survival curves for patients treated with PPV with or without combination chemotherapies. a-f) There was no significant survival advantage of combined chemotherapies (CC) in each intrinsic subtype, compared with the treatment by PPV alone. a) b) mrTNBC: PFS and OS, $P=0.467$ and $P=0.347$, respectively. c,d) luminal/HER2-negative type: PFS and OS, $P=0.220$ and $P=0.850$, respectively. e,f) HER2-positive type: PFS and OS, $P=0.296$ and $P=0.957$, respectively. HER2, human epidermal growth factor receptor 2; mrTNBC, metastatic recurrent triple negative breast cancer; OS, overall survival; PFS, progression-free survival; PPV, personalized peptide vaccination.

with or without increased CTL responses after PPV in each intrinsic subtype. CTL boosting was suggested to be a potential prognostic factor for OS but not for PFS in mrTNBC patients, whereas there was no significant difference between CTL boosting and these prognoses in luminal/HER2-negative and HER2-positive patients (mrTNBC: PFS and OS, $P=0.345$ and $P=0.053$, respectively; luminal/HER2-negative type: PFS and OS, $P=0.272$ and $P=0.740$, respectively; HER2-positive type: PFS and OS, $P=0.714$ and $P=0.758$, respectively).

\section{Discussion}

Since treatment outcomes in mrTNBC patients remain poor [19-21], a novel treatment modality including immunotherapy is required. Several tumor associated antigens (TAAs), such as cancer testis antigens, EGF-R, aldehyde dehydrogenase 1 (ALDH1) and enhancer of zeste homolog 2 (EZH2), are frequently expressed in TNBC, particularly in basal-like subtypes [22-24]. Despite these potential molecular targets for immunotherapy in TNBC, no randomized controlled trials of cancer vaccine have shown promise of clinical benefit to date. We have developed a novel regimen of PPV, in which vaccine antigens are selected and administered from a pool of 31 different peptide candidates based on the pre-existing IgG responses specific to peptides before vaccination [13-17]. In previous studies, PPV was feasible for the vast majority of cancer patients with different HLA-types [13-17]. Based on these results in cancer patients, we conducted a phase II study of PPV for metastatic recurrent breast cancer patients to investigate the feasibility of PPV for mrTNBC. There were no severe adverse events associated with PPV, and most of the mrTNBC patients showed augmented immune responses to PPV.

The current study suggested the feasibility of PPV for mrTNBC patients who had failed standard chemotherapy, since the MPFST and MST of mrTNBC patients were 7.5 and 11.1 months from the first vaccination, respectively. In previously reported studies, the MPFST of mrTNBC patients treated by various chemotherapy and/or targeted therapy regimens was between 2.5 and 6.5 months [7,25-28]. Therefore, the MPFST of 7.5 months in mrTNBC patients treated by PPV in the current study seemed to be promising. Regarding OS in TNBC patients, Dent et al. demonstrated that the MST from recurrence to death was nine months, although the details 


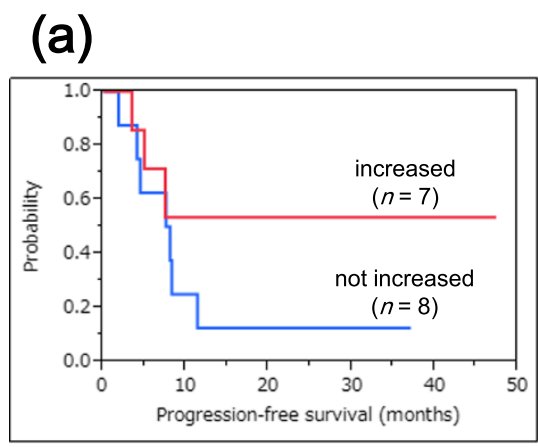

$\operatorname{mrTNBC}$

(b)

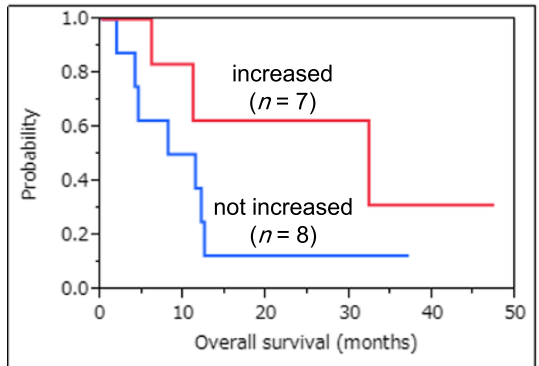

(c)

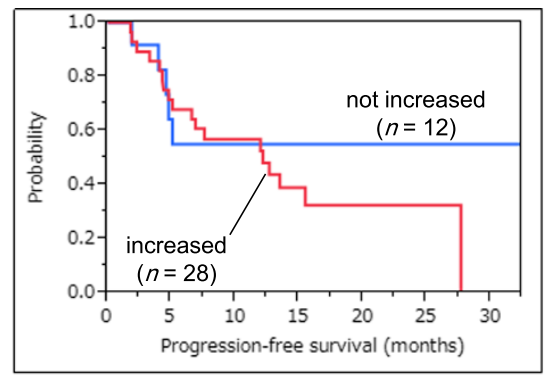

Lum-HER2(-)

(d)

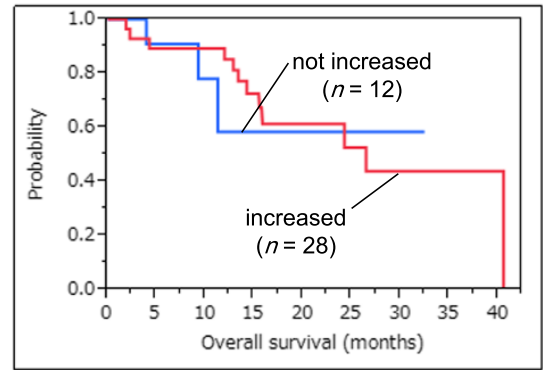

(e)

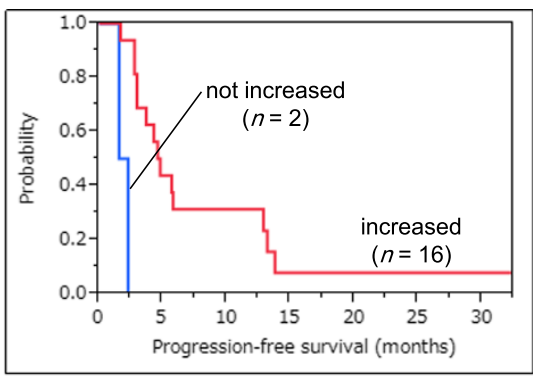

HER2(+)

(f)

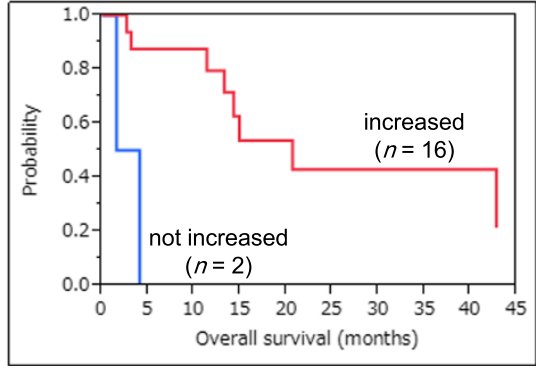

Figure 5 Survival curves for patients with or without increased lgG responses after PPV. a-f) IgG boosting was a significant prognostic factor for OS and PFS in HER2-positive patients, whereas there was no significant difference between increased lgG responses and these prognoses in $\mathrm{mrTNBC}$ and luminal/HER2-negative patients. $\mathbf{a}, \mathbf{b}) \mathrm{mrTNBC}$ : PFS and OS, $P=0.274$ and $P=0.152$, respectively. $\mathbf{c}$, d) luminal/HER2-negative type: PFS and OS, $P=0.732$ and $P=0.571$, respectively. e, f) HER2-positive type: PFS and OS, $P=0.0001$ and $P=0.001$, respectively. HER2, human epidermal growth factor receptor 2; lgG, immunoglobulin G; mrTNBC, metastatic recurrent triple negative breast cancer; OS, overall survival; PFS, progression-free survival; PPV, personalized peptide vaccination.

of chemotherapy regimens were not described [19]. More recently conducted studies showed that the MST of mrTNBC patients treated by various chemotherapy and/ or targeted therapy regimens was between 7.7 and 17.9 months [26-28]. Although almost all patients in these previous studies were enrolled as a first-line and/or second-line treatment [26-28], most patients in the current study were enrolled as a third or more line treatment. In addition, considering that the median duration of previous chemotherapies in the mrTNBC patients in the current study was 9 months, the MST of 11.1 months in mrTNBC patients treated by PPV seems to be encouraging. As a next step, to clarify the clinical benefit of PPV in mrTNBC, we need to conduct a randomized controlled study, in which patients are treated with standard of care (SOC) alone or with PPV plus SOC.

Although the results of immune responses were not significantly different by intrinsic subtypes, a high population of HER2-positive patients showed IgG responses at the sixth vaccination. Since all of the HER2-positive patients had been treated with trastuzumab, antigendependent cellular cytotoxicity might have affected their humoral immunities [29]. Notably, IgG boosting was a significant prognostic factor for OS and PFS in HER2positive patients, although the number of patients was too small to confirm this. Combined chemotherapies also might affect the status of IgG responses, but no survival advantages of combined chemotherapies were shown in our metastatic recurrent breast cancer patients.

The clinical response rate in the present series was 14.0\%, including three CR and six PR cases. Among these responsive patients, combined chemotherapy was used in eight cases and hormonal therapy in one case. The intrinsic subtype of these patients was luminal/ HER2-negative type in seven cases and mrTNBC in two cases. Notably, the number of previous chemotherapy regimens was one regimen in two patients and two regimens in seven patients. In our study, more than four regimens of previous chemotherapy were significantly correlated with poor prognosis (data not shown). From these results, we would recommend PPV within three regimens of previous chemotherapy for metastatic recurrent breast cancer patients. A greater number of previous chemotherapy regimens could not sufficiently enhance 


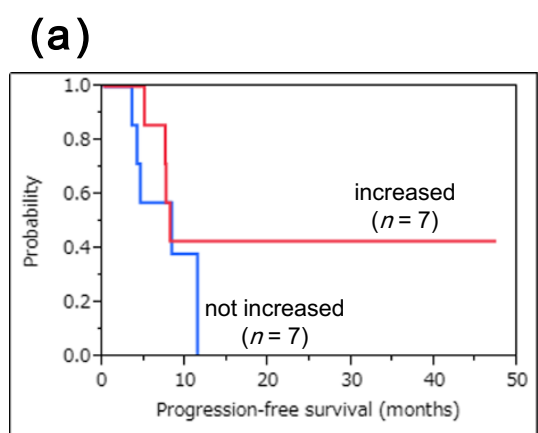

mrTNBC

(b)

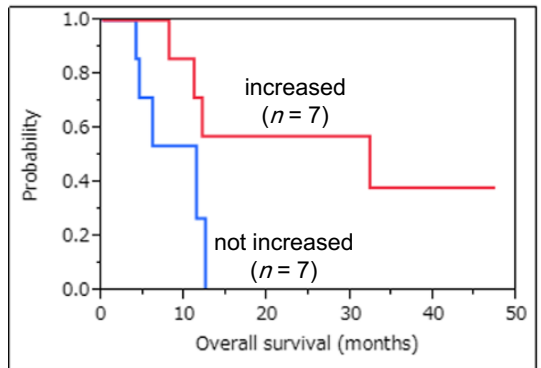

(c)

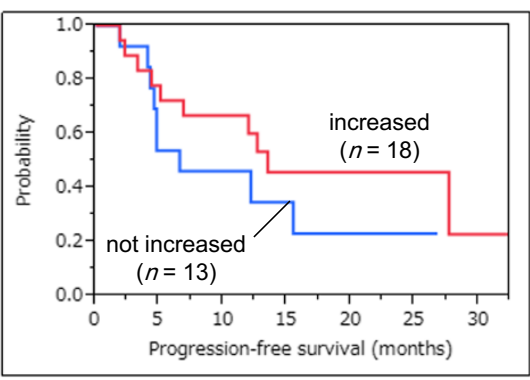

Lum-HER2(-)

(d)

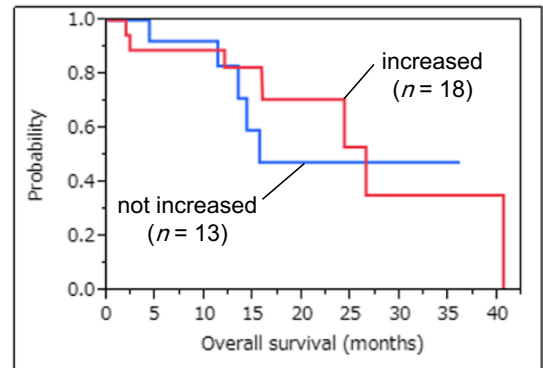

(e)

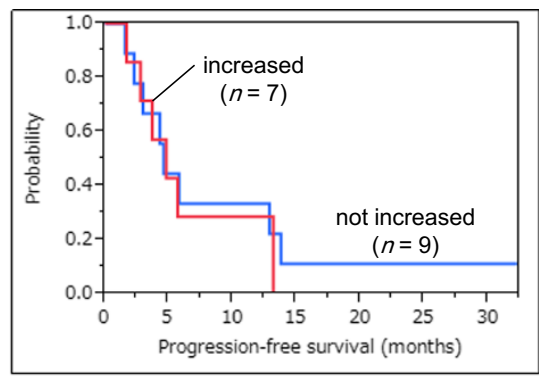

HER2(+) (f)

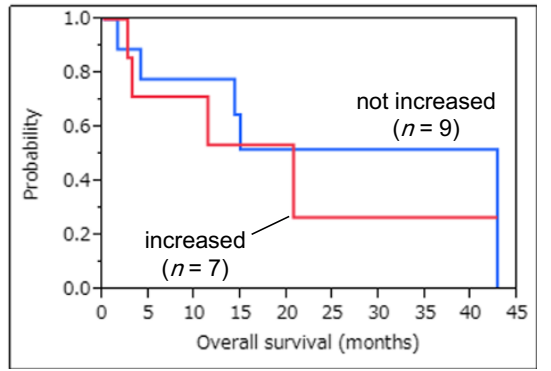

Figure 6 Survival curves for patients with or without increased CTL responses after PPV. a-f) CTL boosting was suggested to be a potential prognostic factor for OS but not for PFS in mrTNBC patients, whereas there was no significant difference between CTL boosting and these prognoses in luminal/HER2-negative and HER2-positive patients. $\mathbf{a}, \mathbf{b}) \operatorname{mrTNBC}$ : PFS and OS, $P=0.345$ and $P=0.053$, respectively. $\mathbf{c}$, d) luminal/HER2-negative type: PFS and OS, $P=0.272$ and $P=0.740$, respectively. e, f) HER2-positive type: PFS and OS, $P=0.714$ and $P=0.758$, respectively. CTL, cytotoxic T lymphocytes; HER2, human epidermal growth factor receptor 2; mrTNBC, metastatic recurrent triple negative breast cancer; OS, overall survival; PFS, progression-free survival; PPV, personalized peptide vaccination.

clinical responses to PPV, particularly in HER2-positive patients. Because of the significant clinical benefit and conventional usage of trastuzumab, the duration of previous chemotherapy was significantly prolonged in HER2positive patients. The status of tumor molecular biology could change and be complicated by this long-term chemotherapy, eventually leading to a poor prognosis. For HER2-positive patients, the induction of PPV should be earlier than that in our HER2-positive patients. Since combined chemotherapies increase the number of severe adverse events in metastatic breast cancer patients, treatment with PPV alone should be performed to maintain their quality of life.

We had an opportunity to confirm the peritumoral infiltration of lymphocytes in the lung metastasis of mrTNBC. Two of the four vaccinated peptide antigens (EGF-R and squamous cell carcinoma antigen recognized by T-cells 2 (SART2)) were expressed in the lung tumor, and CTL responses to the SART2-93 antigen were significantly increased in this case (case 18 in Additional file 2: Table S2). Although IgG responses to the two peptide antigens were not significantly increased, PPV could enhance the anti-tumor immunity and efficacy of combined chemotherapy in this case. We have investigated the expressions of 15 TAAs in primary and recurrent breast cancer tissues by immunohistochemistry (RT, unpublished data). We found that 10 of 15 TAAs were expressed in both primary and recurrent breast cancer tissues, except for lymphocyte specific protein tyrosine kinase (LCK), prostate specific antigen (PSA), prostate specific membrane antigen (PSMA), prostatic acid phosphatase (PAP) and multidrug resistance-associated protein 3 (MRP3). However, four of these five TAAs, including LCK, PSA, PAP and MRP3, were reported to be expressed in breast cancer tissues, although the frequency of expression was lower than that of other TAAs [30-34]. Therefore, 14 of the 15 TAAs could be potential molecular targets for immunotherapy in breast cancer patients.

\section{Conclusions}

In conclusion, PPV could be feasible for mrTNBC patients because of the safety, immune responses and possible clinical benefits. For mrTNBC patients, we are planning a randomized controlled study, in which patients are treated with SOC alone or with PPV plus SOC to further clarify the clinical benefit of PPV. 


\section{Additional files} Additional file 1: Table S1. Information on the peptide candidates
used for PPV.

Additional file 2: Table S2. Immune responses to vaccinated peptides in mrTNBC patients.

Additional file 3: Table S3. Immune responses to vaccinated peptides in luminal/HER2-negative patients.

Additional file 4: Table S4. Immune responses to vaccinated peptides in HER2-positive patients.

\section{Abbreviations}

ALDH1: aldehyde dehydrogenase 1; CR: complete response; CT: computed tomography; CTL: cytotoxic T lymphocyte; ECOG: Eastern Cooperative Oncology Group; EGF-R: epidermal growth factor receptor; EZH2: enhancer of zeste homolog 2; FEC: 5- fluorouracil/epirubicin/cyclosphamide; HER2: human epidermal growth factor 2; INF: interferon; LCK: lymphocyte specific protein tyrosine kinase; MPFST: median progression-free survival time; MRI: magnetic resonance imaging; MRP3: multidrug resistance-associated protein 3; mrTNBC: metastatic recurrent triple-negative breast cancer; MST: median overall survival time; nab-PTX: nanoparticle albumin-bound paclitaxel; NCl-CTC Ver.-3.0: National Cancer Institute common terminology criteria for adverse events version 3.0; OS: overall survival; PAP: prostatic acid phosphatase; PBMCs: peripheral blood mononuclear cells; PD: progressive disease; PFS: progression-free survival; PPV: personalized peptide vaccination; PR: partial response; PSA: prostate specific antigen; PSMA: prostate specific membrane antigen; RECIST: Response Evaluation Criteria in Solid Tumors; SART2: squamous cell carcinoma antigen recognized by T-cells 2; SD: stable disease; SOC: standard of care; TAAs: tumor associated antigens; T-DM1: trastuzumab emtansine; TNBC: triple-negative breast cancer.

\section{Competing interests}

Akira Yamada, is a Board member of the Green Peptide Company, Ltd. Kyogo Itoh and Akira Yamada have stock of the Green Peptide Company, Ltd. Kyogo Itoh received research fund from Taiho Pharmaceutical Company. The other authors declare that they have no competing interests.

\section{Authors' contributions}

RT, UT and Kl are responsible for the conception and design of the study, the acquisition, analysis and interpretation of data, and drafting the work. TS is responsible for the interpretation of data and drafting the work. NI, MT, $\mathrm{HO}, \mathrm{MF}, \mathrm{TF}, \mathrm{NS}, \mathrm{YA}$ and $\mathrm{AY}$ are responsible for the interpretation of data and revising the work critically. AK, MK and SM are responsible for the acquisition and analysis of data and revising the work critically. All authors read and approved the final manuscript.

\section{Acknowledgements}

The authors thank Dr. Hirotaka Iwase (Department of Breast and Endocrine Surgery, Kumamoto University, Kumamoto, Japan) for providing the resected specimens of the SD case (case 18 in Additional file 2: Table S2). This study was supported in part by grants from the Regional Innovation Cluster Program, by the Project for Development of Innovative Research on Cancer Therapeutics (P-Direct) of the Ministry of Education, Culture, Sports, Science and Technology of Japan, and by the Sendai Kousei Hospital.

\section{Author details}

'Department of Surgery, Kurume University School of Medicine, 67 Asahi-machi, Kurume 830-0011, Japan. ${ }^{2}$ Research Center for Innovative Cancer Therapy, Kurume University School of Medicine, 67 Asahi-machi, Kurume 830-0011, Japan. ${ }^{3}$ Department of Pathology, Kurume University School of Medicine, 67 Asahi-machi, Kurume 830-0011, Japan. ${ }^{4}$ Department of Immunology and Immunotherapy, Kurume University School of Medicine, 67 Asahi-machi, Kurume 830-0011, Japan.

Received: 8 October 2013 Accepted: 23 June 2014 Published: 3 July 2014

\section{References}

1. Gradishar WJ, Krasnojon D, Cheporov S, Makhson AN, Manikhas GM, Clawson A, Bhar P: Significantly longer progression-free survival with nab-paclitaxel compared with docetaxel as first-line therapy for metastatic breast cancer. J Clin Oncol 2009, 27:3611-3619.

2. Robinson DM, Keating GM: Albumin-bound Paclitaxel: in metastatic breast cancer. Drugs 2006, 66:941-948.

3. Cortes J, O'Shaughnessy J, Loesch D, Blum JL, Vahdat LT, Petrakova K, Chollet P, Manikas A, Diéras V, Delozier T, Vladimirov V, Cardoso F, Koh H, Bougnoux P, Dutcus CE, Seegobin S, Mir D, Meneses N, Wanders J, Twelves C: Eribulin monotherapy versus treatment of physician's choice in patients with metastatic breast cancer (EMBRACE): a phase 3 open-label randomised study. Lancet 2011, 377:914-923.

4. Twelves C, Cortes J, Vahdat LT, Wanders J, Akerele C, Kaufman PA: Phase III trials of eribulin mesylate (E7389) in extensively pretreated patients with locally recurrent or metastatic breast cancer. Clin Breast Cancer 2010, 10:160-163.

5. Miller K, Wang M, Gralow J, Dickler M, Cobleigh M, Perez EA, Shenkier T, Cella D, Davidson NE: Paclitaxel plus bevacizumab versus paclitaxel alone for metastatic breast cancer. N Engl J Med 2007, 357:2666-2676.

6. Miles DW, Chan A, Dirix LY, Cortés J, Pivot X, Tomczak P, Delozier T, Sohn $J H$, Provencher L, Puglisi F, Harbeck N, Steger GG, Schneeweiss A, Wardley AM, Chlistalla A, Romieu G: Phase III study of bevacizumab plus docetaxel compared with placebo plus docetaxel for the first-line treatment of human epidermal growth factor receptor 2-negative metastatic breast cancer. J Clin Oncol 2010, 28:3239-3247.

7. Robert NJ, Diéras V, Glaspy J, Brufsky AM, Bondarenko I, Lipatov ON, Perez EA, Yardley DA, Chan SY, Zhou X, Phan SC, O'Shaughnessy J: RIBBON-1: randomized, double-blind, placebo-controlled, phase III trial of chemotherapy with or without bevacizumab for first-line treatment of human epidermal growth factor 2-negative, locally recurrent or metastatic breast cancer. J Clin Oncol 2011, 29:1252-1260.

8. Di Leo A, Jerusalem G, Petruzelka L, Torres R, Bondarenko IN, Khasanov R, Verhoeven D, Pedrini JL, Smirnova I, Lichinitser MR, Pendergrass K, Garnett S, Lindemann JP, Sapunar F, Martin M: Results of the CONFIRM phase III trial comparing fulvestrant $250 \mathrm{mg}$ with fulvestrant $500 \mathrm{mg}$ in postmenopausal women with estrogen receptor-positive advanced breast cancer. J Clin Oncol 2010, 28:4594-4600.

9. Mehta RS, Barlow WE, Albain KS, Vandenberg TA, Dakhil SR, Tirumali NR, Lew DL, Hayes DF, Gralow JR, Livingston RB, Hortobagyi GN: Combination anastrozole and fulvestrant in metastatic breast cancer. $N$ Engl J Med 2012, 367:435-444.

10. Baselga J, Swain SM: CLEOPATRA: a phase III evaluation of pertuzumab and trastuzumab for HER2-positive metastatic breast cancer. Clin Breast Cancer 2010, 10:489-491.

11. Baselga J, Cortés J, Kim SB, Im SA, Hegg R, Im YH, Roman L, Pedrini JL, Pienkowski T, Knott A, Clark E, Benyunes MC, Ross G, Swain SM, CLEOPATRA Study Group: Pertuzumab plus trastuzumab plus docetaxel for metastatic breast cancer. N Engl J Med 2012, 366:109-119.

12. Verma S, Miles D, Gianni L, Krop IE, Welslau M, Baselga J, Pegram M, Oh DY, Diéras V, Guardino E, Fang L, Lu MW, Olsen S, Blackwell K, EMILIA Study Group: Trastuzumab emtansine for HER2-positive advanced breast cancer. N Engl J Med 2012, 367:1783-1791.

13. Sasada T, Komatsu N, Suekane S, Yamada A, Noguchi M, Itoh K: Overcoming the hurdles of randomized clinical trials of therapeutic cancer vaccines. Euro J Cancer 2010, 46:1514-1519.

14. Terasaki M, Shibui S, Narita Y, Fujimaki T, Aoki T, Kajiwara K, Sawamura Y, Kurisu K, Mineta T, Yamada A, Itoh K: Phase I trial of a personalized peptide vaccine for patients positive for human leukocyte antigen-A24 with recurrent or progressive glioblastoma multiforme. J Clin Oncol 2011, 29:337-344.

15. Yanagimoto $H$, Shiomi $H$, Satoi $S$, Mine $T$, Toyokawa $H$, Yamamoto $T$, Tani $T$, Yamada A, Kwon AH, Komatsu N, Itoh K, Noguchi M: A phase II study of personalized peptide vaccination combined with gemcitabine for non-resectable pancreatic cancer patients. Oncol Rep 2010, 24:795-801.

16. Hattori T, Mine T, Komatsu N, Yamada A, Itoh K, Shiozaki H, Okuno K: Immunological evaluation of personalized peptide vaccination in combination with UFT and UZEL for metastatic colorectal carcinoma patients. Cancer Immunol Immunother 2009, 58:1843-1852.

17. Noguchi M, Mine T, Komatsu N, Suekane S, Moriya F, Matsuoka K, Yutani S, Shichijo S, Yamada A, Toh U, Kawano K, Azuma K, Uemura H, Okuno K, 
Matsumoto K, Yanagimoto H, Yamanaka R, Oka M, Todo S, Sasada T, Itoh K: Assessment of immunological biomarkers in patients with advanced cancer treated by personalized peptide vaccination. Cancer Biol Ther 2011, 10:1266-1279.

18. Komatsu N, Shichijo S, Nakagawa M, Itoh K: New multiplexed flow cytometric assay to measure anti-peptide antibody: a novel tool for monitoring immune responses to peptides used for immunization. Scand J Clin Lab Invest 2004, 64:535-545.

19. Dent R, Trudeau M, Pritchard Kl, Hanna WM, Kahn HK, Sawka CA, Lickley LA, Rawlinson E, Sun P, Narod SA: Triple-negative breast cancer: clinical features and patterns of recurrence. Clin Cancer Res 2007, 13:4429-4434.

20. Kassam F, Enright K, Dent R, Dranitsaris G, Myers J, Flynn C, Fralick M, Kumar R, Clemons M: Survival outcomes for patients with metastatic triple-negative breast cancer; implications for clinical practice and trial design. Clin Breast Cancer 2009, 9:29-33.

21. Foulkes WD, Smith IE, Reis-Filho JS: Triple-negative breast cancer. N Engl J Med 2010, 363:1938-1948.

22. Curigliano G, Viale G, Ghioni M, Jungbluth AA, Bagnardi V, Spagnoli GC, Neville AM, Nolè F, Rotmensz N, Goldhirsch A: Cancer-testis antigen expression in triple-negative breast cancer. Ann Oncol 2011 22:98-103.

23. Ueno NT, Zhang D: Targeting EGFR in triple negative breast cancer. J Cancer 2011, 2:324-328.

24. De Brot M, Rocha RM, Soares FA, Gobbi H: Prognostic impact of the cancer stem cell related markers ALDH1 and EZH2 in triple negative and basal-like breast cancers. Pathology 2012, 44:303-312.

25. Finn RS, Press MF, Dering J, Arbushites M, Koehler M, Oliva C, Williams LS, Di Leo A: Estrogen receptor, progesterone receptor, human epidermal growth factor receptor 2 (HER2), and epidermal growth factor receptor expression and benefit from lapatinib in a randomized trial of paclitaxel with lapatinib or placebo as first-line treatment in HER2-negative or unknown metastatic breast cancer. J Clin Oncol 2009, 27:3908-3915.

26. O'Shaughnessy J, Osborne C, Pippen JE, Yoffe M, Patt D, Rocha C, Koo IC, Sherman BM, Bradley C: Iniparib plus chemotherapy in metastatic triple-negative breast cancer. N Engl J Med 2011, 364:205-214

27. Brufsky A, Valero V, Tiangco B, Dakhil S, Brize A, Rugo HS, Rivera R, Duenne A, Bousfoul N, Yardley DA: Second-line bevacizumabcontaining therapy in patients with triple-negative breast cancer: subgroup analysis of the RIBBON-2 trial. Breast Cancer Res Treat 2012, 133:1067-1075

28. Baselga J, Segalla JG, Roché $H$, Del Giglio A, Pinczowski $H$, Ciruelos EM, Filho SC, Gómez P, Van Eyll B, Bermejo B, Llombart A, Garicochea B, Durán MÁ Hoff PM, Espié M, de Moraes AA, Ribeiro RA, Mathias C, Gil Gil M, Ojeda B, Morales J, Kwon Ro S, Li S, Costa F: Sorafenib in combination with capecitabine: an oral regimen for patients with HER2-negative locally advanced or metastatic breast cancer. J Clin Oncol 2012, 30:1484-1491.

29. Musolino A, Naldi N, Bortesi B, Pezzuolo D, Capelletti M, Missale G, Laccabue D, Zerbini A, Camisa R, Bisagni G, Neri TM, Ardizzoni A: Immunoglobulin $\mathrm{G}$ fragment $\mathrm{C}$ receptor polymorphisms and clinical efficacy of trastuzumab-based therapy in patients with HER-2/neu-positive metastatic breast cancer. J Clin Oncol 2008, 26:1789-1796.

30. Elsberger B, Fullerton R, Zino S, Jordan F, Mitchell TJ, Brunton VG, Mallon EA, Shiels PG, Edwards J: Breast cancer patients' clinical outcome measures are associated with Src kinase family member expression. Br J Cancer 2010, 103:899-909.

31. Narita D, Raica M, Suciu C, Cîmpean A, Anghel A: Immunohistochemical expression of androgen receptor and prostate-specific antigen in breast cancer. Folia Histochem Cytobiol 2006, 44:165-172.

32. Wang Y, Harada M, Yano H, Ogasawara S, Takedatsu H, Arima Y, Matsueda S, Yamada A, Itoh K: Prostatic acid phosphatase as a target molecule in specific immunotherapy for patients with nonprostate adenocarcinoma. $J$ Immunother 2005, 28:535-541.
33. Partanen L, Staaf J, Tanner M, Tuominen VJ, Borg Å, Isola J: Amplification and overexpression of the $A B C C 3$ (MRP3) gene in primary breast cancer. Genes Chromosomes Cancer 2012, 51:832-840.

34. Chang SS, Reuter VE, Heston WD, Bander NH, Grauer LS, Gaudin PB: Five different anti-prostate-specific membrane antigen (PSMA) antibodies confirm PSMA expression in tumor-associated neovasculature. Cancer Res 1999, 59:3192-3198.

doi:10.1186/bcr3685

Cite this article as: Takahashi et al:: Feasibility study of personalized peptide vaccination for metastatic recurrent triple-negative breast cancer patients. Breast Cancer Research 2014 16:R70.

\section{Submit your next manuscript to BioMed Central and take full advantage of:}

- Convenient online submission

- Thorough peer review

- No space constraints or color figure charges

- Immediate publication on acceptance

- Inclusion in PubMed, CAS, Scopus and Google Scholar

- Research which is freely available for redistribution

Submit your manuscript at www.biomedcentral.com/submit
C BioMed Central 